\title{
Inflamed Tumor Phenotype as Predictor of Long-Term Response to Pembrolizumab in an EGFR-Mutated Non- Small Cell Lung Cancer (NSCLC) Patient with Acquired Resistance to Afatinib: a Case Report and Review of the Literature
}

\author{
Sara Baglivo · Martina Mandarano · Guido Bellezza • Vincenzo Minotti • \\ Angelo Bonaiti · Matthias J. Fischer · Ilaria Birocchi · Fausto Roila • \\ Niccolò Metelli · Vienna Ludovini · Giulio Metro (iD
}

Received: December 1, 2021 / Accepted: January 10, 2022 / Published online: January 23, 2022

(C) The Author(s) 2022

\section{ABSTRACT}

Treatment with immune checkpoint inhibitors (ICIs) that target the programmed cell death 1/programmed cell death ligand-1 (PD-1/PD-L1) axis is usually ineffective in patients with epidermal growth factor receptor (EGFR)-mutated advanced non-small cell lung cancer (NSCLC), either as first-line treatment or in later lines. By contrast, especially for patients with common EGFR mutations (exon 19 deletion/L858R point mutation), an orally bioavailable EGFR tyrosine kinase inhibitor (EGFR-TKI) is the best upfront therapy, being able to provide response rates well above $50 \%$ and a median progression-free

S. Baglivo - V. Minotti - A. Bonaiti - I. Birocchi · N. Metelli · V. Ludovini · G. Metro ( $\varangle)$

Medical Oncology, Santa Maria della Misericordia Hospital, Azienda Ospedaliera di Perugia, Perugia, Italy

e-mail: giulio.metro@ospedale.perugia.it

M. Mandarano · G. Bellezza

Section of Anatomic Pathology and Histology, Department of Medicine and Surgery, University of Perugia, Perugia, Italy

\section{J. Fischer}

Department of Radiology, Santa Maria della Misericordia Hospital, Azienda Ospedaliera di Perugia, Perugia, Italy

F. Roila

Medical Oncology, Santa Maria della Misericordia

Hospital, University of Perugia, Perugia, Italy survival ranging from 11 to 19 months, depending on whether a second-generation (e.g., afatinib) or a third-generation (i.e., osimertinib) EGFR-TKI is used. Unfortunately, treatment options for these patients at the time of acquired resistance are limited. As for afatinib-pretreated patients, those who develop a T790M mutation may benefit from osimertinib, whereas platinum-based chemotherapy is the preferable therapeutic strategy for T790M-negative patients as well as for patients who progress on osimertinib administered as first-line therapy. Here, we describe the case of an exon19-deleted patient who experienced a complete response to the anti-PD-1 agent pembrolizumab upon the development of T790M-negative acquired resistance to afatinib. Furthermore, we discuss this case in the context of the existing literature, especially focusing on the importance of evaluating multiple markers of immune response post-EGFR-TKI and prior to ICI treatment in order to select the best treatment strategy in this clinical scenario.

Keywords: Afatinib; EGFR mutation; EGFR-TKI, immune checkpoint inhibitors; PD-L1; Tumor mutation burden 


\section{Key Summary Points}

Treatment with immune checkpoint inhibitors is generally poorly effective for epidermal growth factor receptor (EGFR)-mutated non-small cell lung cancer (NSCLC) patients who experience acquired resistance to an EGFR-tyrosine kinase inhibitor (TKI).

Here, we present the case of an EGFRmutated NSCLC patient who underwent a complete response to the antiprogrammed cell death 1 (PD-1) agent pembrolizumab upon disease progression on the EGFR-TKI afatinib.

Emphasis is placed on the serial measurement of multiple immune markers in tumor tissue as potential predictors of response to immunotherapy.

In our case, programmed cell death ligand1 (PD-L1) expression, tumor mutation burden, as well as the presence/absence of CD8+ and FOXP3+ Tregs tumor-

infiltrating lymphocytes were useful markers for the existence of an inflamed phenotype.

Evaluating the aforementioned markers can help guide treatment decisions on whether EGFR-mutated NSCLCs with acquired resistance to an EGFR-TKI could benefit from immunotherapy.

\section{INTRODUCTION}

Treatment with an epidermal growth factor receptor tyrosine kinase inhibitor (EGFR-TKI) is highly effective for EGFR-mutated advanced non-small cell lung cancer [1]. However, limited treatment options exist at the time of acquired resistance to an EGFR-TKI, especially for T790M-negative patients who progress on afatinib and for those who receive osimertinib as a first-line therapy. Unfortunately, despite being

extensively studied in clinical trials, immunotherapy with immune checkpoint inhibitors (ICIs) that target the programmed cell death 1/programmed cell death ligand-1 (PD-1/PD-L1) axis has been shown to be poorly effective in the aforementioned post-EGFR-TKI clinical scenario [2]. Here we present the case of an EGFR-mutated (exon 19 deletion) advanced NSCLC patient who underwent a rapid complete response to the anti-PD-1 agent pembrolizumab used right after the development of T790M-negative acquired resistance to afatinib. The case is discussed in light of the existing literature on the basis of serial biopsies that were performed over time in order to measure potential markers of immune response, with the ultimate aim of predicting the clinical benefit of ICI treatment.

\section{CASE}

A 61-year-old woman who had never smoked was diagnosed with resectable stage III adenocarcinoma of the left lung. The patient was initially treated with three cycles of neoadjuvant cisplatin/gemcitabine followed by pneumonectomy. However, no pathologic response was documented at histologic examination, which confirmed the presence of stage IIIA (pN2) adenocarcinoma. Three months later, a CT scan documented progression in the liver for the presence of a single metastasis that was surgically resected. Unfortunately, the disease progressed again within 2 months following the hepatic metastasectomy, as documented by the development of multiple nodules in the right lung and a single asymptomatic brain lesion. Next-generation sequencing (NGS) (Ion Ampliseq Colon and Lung Research Panel v.2 and Ion GeneStudio S5 Systems, Thermo Fisher Scientific) showed the presence of an exon 19 deletion in the EGFR gene in both the primary lung tumor and hepatic metastasis (Fig. 1A, B), with a PD-L1 tumor proportion score (TPS) at immunohistochemistry of $60 \%$ (clone 22C3, pharmDx kit, Dako) in both samples (Fig. 2A, D). On this basis, the patient started treatment with the EGFR tyrosine kinase inhibitor (TKI) afatinib, which led to a partial response in the 


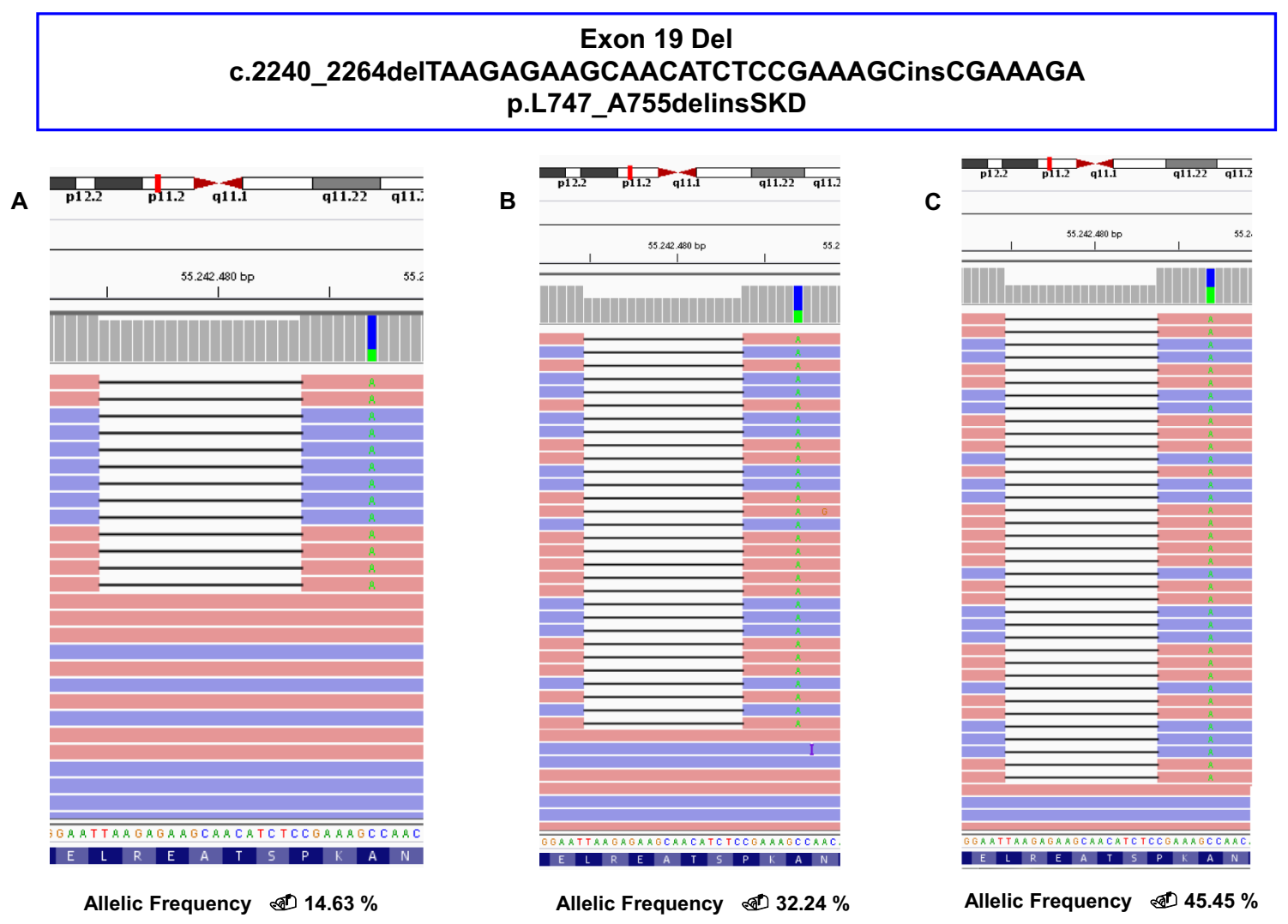

Fig. 1 Next-generation sequencing (NGS) performed on the primary lung tumor at the time of first diagnosis (A), on the hepatic metastasis prior to the start of treatment with afatinib (B), and on a mediastinal lymph node at the time of acquired resistance to afatinib before the start of

lung and in the brain. Nevertheless, 8 months after the initiation of afatinib, the disease progressed further in the mediastinal lymph nodes, right lung, and brain (Fig. 3A, D, G). Re-biopsy was performed on the right lower paratracheal lymph-node station, which confirmed the presence of adenocarcinoma with an EGFR exon 19 deletion at NGS and without the presence of the secondary $\mathrm{T} 790 \mathrm{M}$ resistance mutation (Fig. 1C). In this sample, a maintained PD-L1 TPS of $60 \%$ was found (Fig. 2G). At that time, all three tissue samples of the patient were further analyzed for tumor mutation burden (TMB) using the Oncomine Tumor Mutation Load (TML) Assay on the Ion GeneStudio S5 System (Thermo Fisher Scientific) (Fig. 4), as well as for tumor-infiltrating lymphocytes (TILs), particularly CD8+ T cell infiltrates and FOXP3+ regulatory $\mathrm{T}$ cells (Tregs) by immunohistochemistry immunotherapy (C). The presence of an EGFR exon 19 deletion was consistently shown in each tumor sample. No T790M mutation was documented in the mediastinal lymph-node sample collected at the time of acquired resistance to afatinib

(Fig. 2B, E, H, and C, F, I, respectively). On the one hand, although low, TMB levels (defined as the total number of nonsynonymous mutations within a tumor genome/per megabase $(\mathrm{Mb})$ ) were found to increase over time (Fig. 4). On the other, the TILs presented both a peripheral and, although with lower density, an intratumoral localization (not evaluable in the re-biopsy mediastinal lymph-nodal specimen), showing high levels of CD8+ T cell infiltrates with scant presence of FOXP3+ Tregs across all tumor samples, which suggested the presence of an inflamed tumor phenotype. On this basis, the patient started pembrolizumab $200 \mathrm{mg}$ i.v. every three weeks, with a first CT scan performed after 2 months of treatment showing the achievement of a partial response at all sites, brain included (Fig. 3B, E, H). Subsequent CT scans showed that the disease continued to 

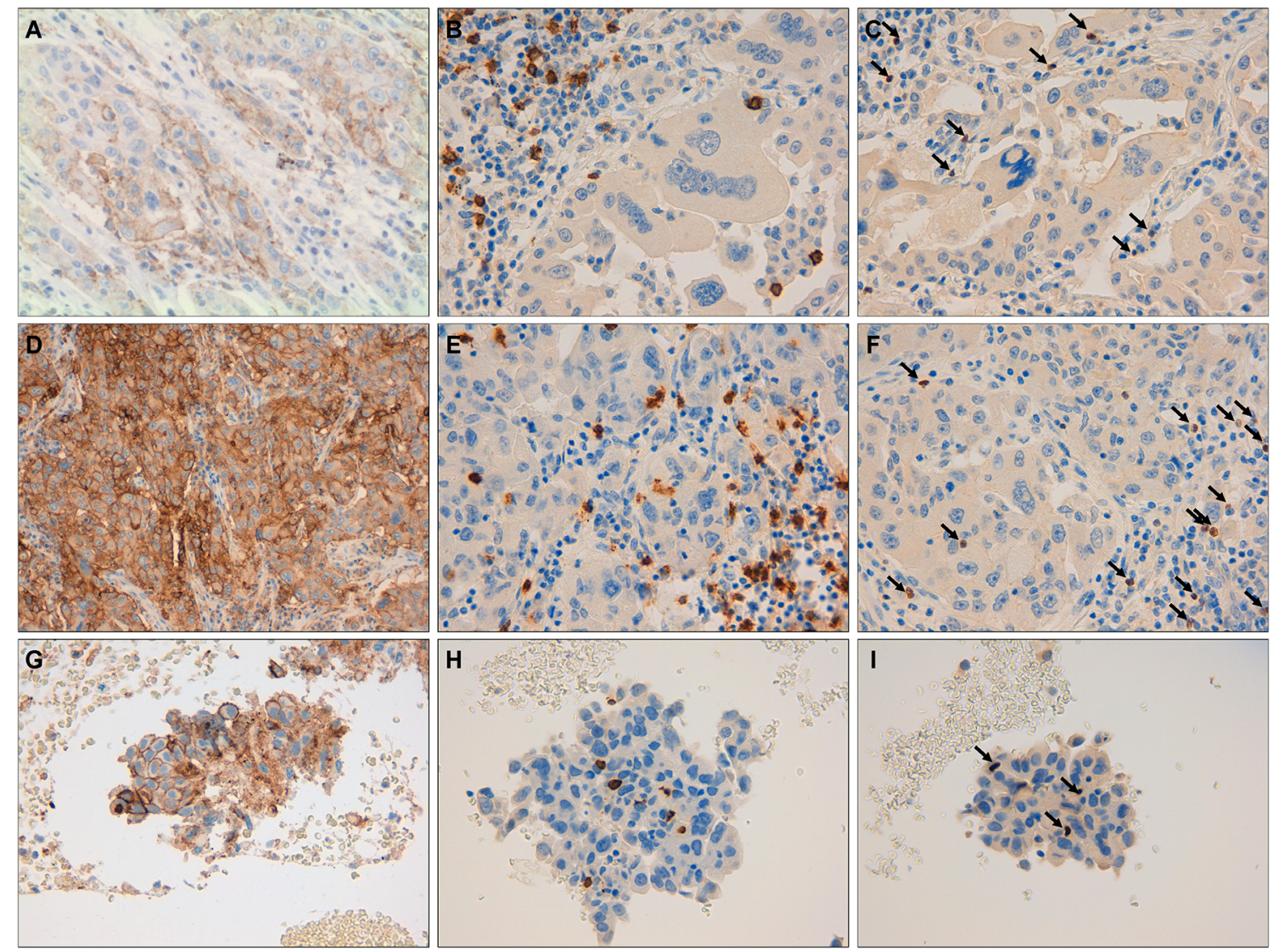

Fig. 2 PD-L1 membranous immunostaining of $60 \%$ of tumor cells, showing high intensity of immunolabeling in the primary lung tumor $(\mathbf{A})$, hepatic metastasis $(\mathbf{D})$, and mediastinal lymph node (G). CD8+ $\mathrm{T}$ cell infiltrates highlighted by membranous immunostaining (brownish label): localized at the edge and between the tumor cells for

shrink until it underwent a complete response after 9 months of treatment (Fig. 3C, F, I). Maintenance of the complete response was observed at the most recent CT scan performed 24 months since the initiation of pembrolizumab.

Written informed consent was obtained from the patient before publishing her case in an anonymous form.

\section{DISCUSSION}

ICI therapy is generally poorly effective for EGFR-mutated NSCLC. Two pooled meta-analyses evaluating a few trials of treatment with both primary lung carcinoma (B) and hepatic metastasis (E) and within the tumor cluster for the nodal relapse $(\mathbf{H})$. scant FOXP3 nuclear immunostaining of Treg cells (arrows) in the primary lung carcinoma (C), hepatic metastasis $(\mathbf{F})$, and nodal relapse $(\mathbf{I})$. Original magnification A-I: $\times 400$

PD-1/PD-L1 inhibitors as a second-line therapy for advanced NSCLC found that immunotherapy does not improve survival as compared to chemotherapy $[3,4]$. Similarly, poor activity of ICI therapy in EGFR-mutated NSCLC has also been reported in a large retrospective analysis by Mazeries et al., in which a response rate of $12.2 \%$ was observed in the subset of patients harboring an EGFR mutation [5]. On the other hand, several phase 1 trials exploring the association of ICI with an EGFR-TKI found that this combination resulted into a high percentage of treatment-related adverse events, which discouraged further clinical development of such a strategy [6-8]. For instance, the phase 1b 

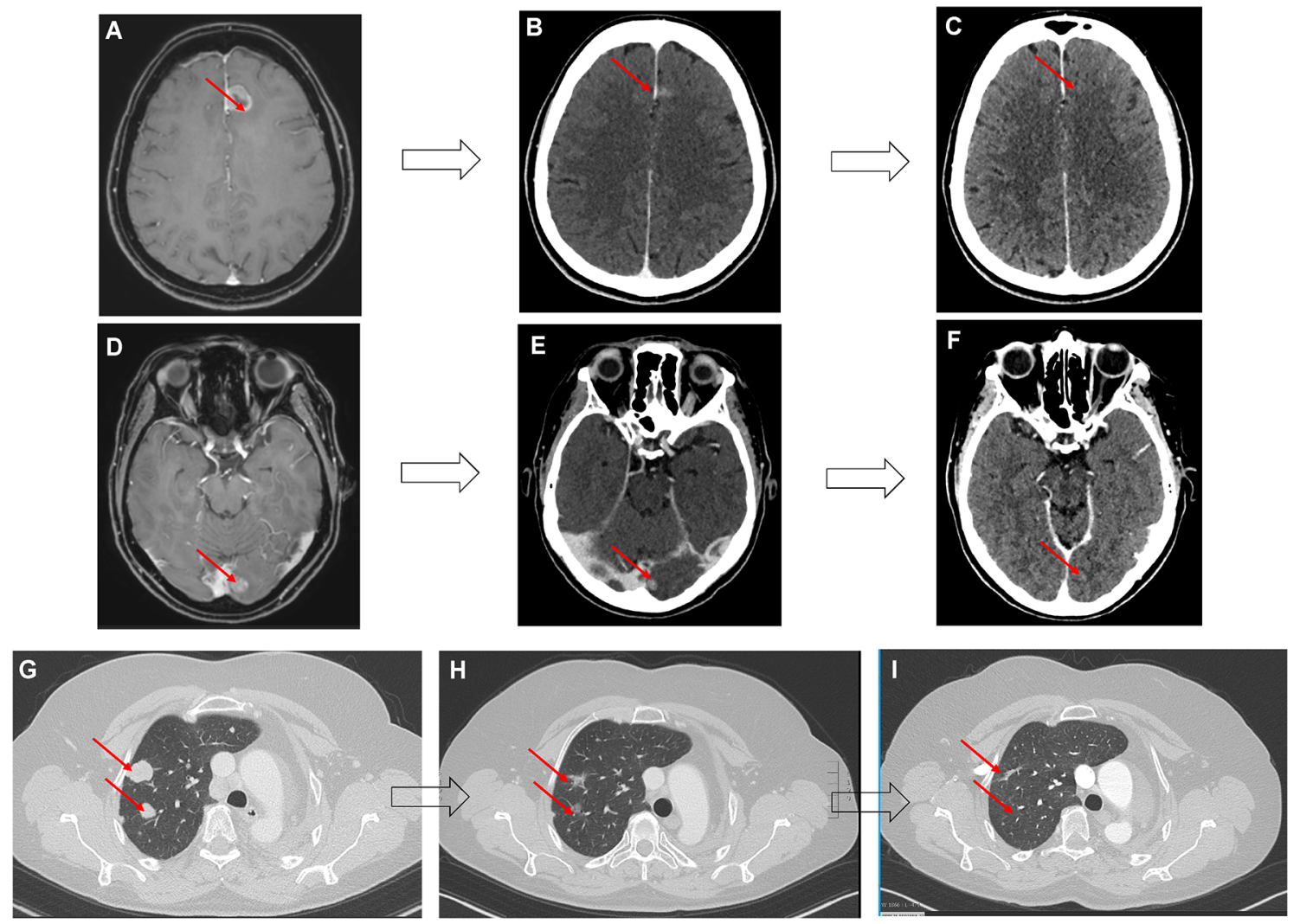

Fig. 3 MRI of the brain (T1 gad sequence) and CT scan of the lung performed right before the start of immunotherapy, showing two centimetric metastatic lesions in the left frontal and occipital lobes $(\mathbf{A}, \mathbf{D})$ and pulmonary metastases in the right lung $(\mathbf{G})$; CT scan of the brain and the lung performed after 2 months of

TATTON study, which evaluated the safety and tolerability of osimertinib plus the anti-PD-L1 agent durvalumab in EGFR-mutated NSCLCs who progressed on prior TKI, documented an unacceptable $38 \%$ incidence of interstitial lung disease (ILD) [6]. These disappointing results imply that the role of ICI therapy for the treatment of patients with EGFR-mutated NSCLC is limited, being confined to patients who progress on an EGFR-TKI and for which no other effective therapies are present.

On the other hand, the fact that occasional responses to immunotherapy have still been reported suggests that proper patient selection is needed to identify the EGFR-mutated NSCLCs who could benefit from ICI therapy. Table 1 shows the anti-tumor response in patients with treatment with pembrolizumab, showing reductions in the sizes of brain lesions $(\mathbf{B}, \mathbf{E})$ and pulmonary metastases $(\mathbf{H})$; CT scan of the brain and the lung performed 9 months after the initiation of pembrolizumab, showing a complete response in the brain $(\mathbf{C}, \mathbf{F})$ and lung $(\mathbf{I})$

an EGFR mutation treated in clinical trials employing anti-PD-1/PD-L1 agents either as monotherapy or in combination with CTLA-4 blockade. Although responses were highly variable, they did not exceed $14 \%$ in the trials that evaluated more than 10 patients. However, it is important to note that firm conclusions cannot be drawn for this clinical scenario given the generally low number of patients that have been included in these studies as well as the heterogeneous types of EGFR mutations (sensitizing common and uncommon and non-sensitizing) that were considered (Table 1).

Importantly, given the impressive and wellestablished activity of targeted therapy in the first-line setting of EGFR-mutated NSCLC, the question of whether immunotherapy should be 


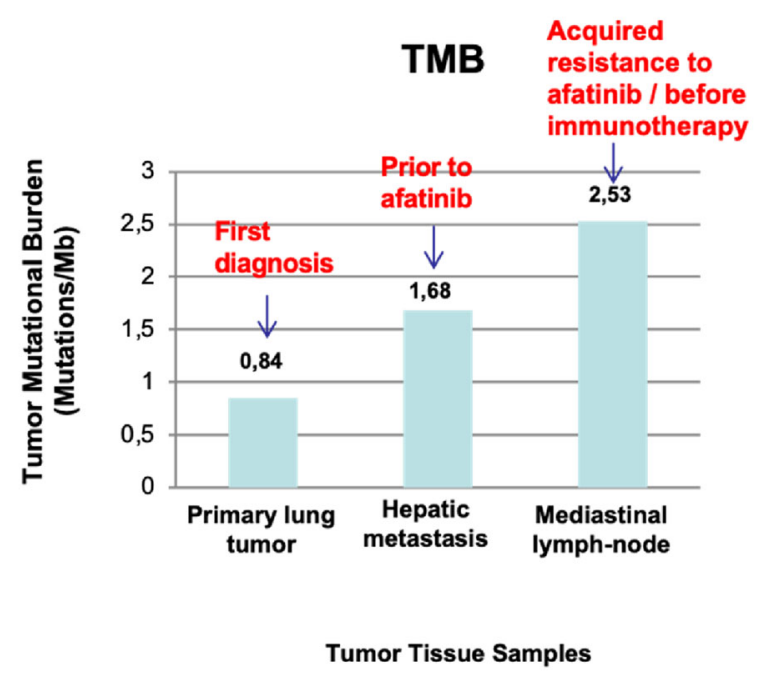

Fig. 4 Assessment of tumor mutation burden (TMB) on the primary lung tumor at the time of the first diagnosis, on hepatic metastasis prior to the start of treatment with afatinib, and on a mediastinal lymph node at the time of acquired resistance to afatinib before the start of immunotherapy. TMB levels, defined as the total number of nonsynonymous mutations within a tumor genome/per megabase $(\mathrm{Mb})$, were progressively increased at the times of first and second disease progression (1.68 and 2.53 mutations $/ \mathrm{Mb}$, respectively) as compared to surgery (0.84 mutations/Mb) administered to patients with an EGFR mutation is particularly relevant for those individuals who develop acquired resistance to an EGFRTKI (i.e., response or stable disease for $\geq 6$ months with subsequent development of disease progression while still on EGFR-TKI). In fact, limited treatment options exist for these patients, and the true effectiveness of immunotherapy is uncertain.

Our case is exceptional, since the patient experienced a deep and durable response (brain included) to ICI therapy despite having a sensitizing EGFR mutation. According to a study, only $2 \%$ of patients with an EGFR sensitizing mutation treated with anti-PD1/PD-L1 agents achieve a long-term response (defined as a complete or partial response lasting for more than 24 months) [16]. The constantly high PDL1 levels expressed by the tumor of the patient $(60 \%)$, even at the time of progression on afatinib, were crucial, along with the demonstration of T790M-negative acquired resistance, for selecting pembrolizumab as the next line of therapy following afatinib. Importantly, while de novo PD-L1 expression is commonly present in EGFR-mutated NSCLCs, likely reflecting a constitutive activation rather than a marker of

Table 1 Activity of ICI therapy in EGFR-mutated advanced NSCLC patients treated in clinical trials with either an antiPD-1/PD-L1 as monotherapy or in combination with CTLA-4 blockade

\begin{tabular}{llcc}
\hline Trial & Drug & Overall response & \\
\cline { 3 - 4 } & & EGFR-TKI naïve & EGFR-TKI pretreated \\
\hline Garon et al. [9] & Pembrolizumab & 2 out of 3 pts $-67 \%$ & 1 out of 26 pts $-4 \%$ \\
Lisberg et al. [10] & Pembrolizumab & 0 out 11 pts $-0 \%^{*}$ & - \\
Gettinger et al. [11] & Nivolumab & 1 out of 7 pts $-14 \%^{* *}$ & \\
Peters et al. [12] & Atezolizumab & 4 out of 45 pts $-9 \%^{* *}$ & \\
Garassino et al. [13] & Durvalumab & & 9 out 64 pts $-14 \%^{* * *}$ \\
Hellmann et al. [14] & Nivolumab + ipilimumab & 4 out of 8 pts $-50 \%^{* *}$ & \\
Gubens et al. [15] & Pembrolizumab + ipilimumab & & 1 out of 10 pts $-10 \%$ \\
\hline
\end{tabular}

EGFR-TKI epidermal growth factor-tyrosine kinase inhibitors, pts patients

${ }^{*} \mathrm{PD}-\mathrm{L} 1 \geq 1 \%$

"Data on pretreatment with an EGFR-TKI are not available

${ }^{* * *} \mathrm{PD}-\mathrm{L} 1 \geq 25 \%$ 
adaptive immune response, high levels of PD-L1 expression at the time of acquired resistance to EGFR-TKI treatment might imply true dependence on the PD-1/PD-L1 axis as an escape mechanism [17-21]. Therefore, PD-L1 reassessment through re-biopsy at the time of acquired resistance to an EGFR-TKI could be warranted in EGFR-mutated patients with acquired resistance to an EGFR-TKI, as this might have important clinical implications. With regard to this, the importance of reassessing PD-L1 expression after EGFR-TKI treatment was elegantly shown by Isomoto et al. in a retrospective study [21]. They demonstrated that 138 EGFR-mutated patients who underwent re-biopsy at the time of acquired resistance to an EGFR-TKI showed a significant increase in PD-L1 expression $\geq 50 \%$ from 14 to $28 \%$. Even more importantly, a higher PD-L1 expression appeared to correlate with an improved clinical outcome in patients who received subsequent ICI treatment.

Tumor mutation burden is another putative marker of sensitivity to immunotherapy [22]. In fact, a higher number of DNA mutations is associated with an increased probability of successfully presenting neoantigens, which, in turn, induce tumor immunogenicity [23]. Importantly, TMB status does not overlap with PD-L1 expression, and assessing both markers could help increase the identification of patients who are candidates for ICI treatment [24]. However, EGFR-mutated patients tend to have lower levels of TMB as compared with $E G F R$ wild-type patients, which is particularly true for patients who bear a common EGFR mutation (exon 19 deletion/L858R point mutation) [25]. This is because EGFR-mutated NSCLC is associated with less tobacco exposure as compared to NSCLC of smokers, and a significant relationship has been demonstrated between smoking history and TMB in advanced lung adenocarcinoma [26]. In addition, the narrow range of TMB levels of expression in EGFR-mutated patients confirms that it may not be a useful biomarker for a therapeutic decision on ICI treatment in this molecularly selected subgroup of patients [25]. Notwithstanding, similarly to other authors, we found that TMB levels increased at the time of acquired resistance to afatinib in our patient [21]. Therefore, although current data suggest that TMB is devoid of predictive significance in EGFR-mutated NSCLC, we still believe that its reassessment in conjunction with PD-L1 measurement in the post-EGFR-TKI setting could help clinical decision making.

Importantly, several other mechanisms than those previously mentioned could be put forward in order to explain the poor sensitivity of EGFR-mutated tumors to immunotherapy. Among others, high levels of the CD73 enzyme, which fosters the immunosuppressive adenosine pathway, as well as the upregulation of genes that belong to the CD73/adenosine pathway can be present in EGFR-mutated tumors [27, 28]. Likewise, CD39, another protein involved in the metabolism of tumorspecific CD8+ $\mathrm{T}$ cells, is generally found at lower levels in EGFR-mutated tumors [29, 30]. However, the presence of an immune desert tumor microenvironment in NSCLCs with EGFR mutations is probably the most common finding. In a study, it was demonstrated that EGFRmutated tumors are characterized by the absence of T-cell infiltration and the presence of a poorly immunogenic tumor microenvironment with a higher proportion of PD-L1+ / CD8+ TILs compared to the EGFR wild-type genotype [31]. By contrast, CD8+ TILs were found to be consistently high in our case, with both a peripheral and intratumoral distribution, which, added to a high expression of PD-L1, suggest that the tumor should be classified as inflamed, resulting in a neoplasm that is more likely responsive to ICI therapies [32]. On the other hand, Tregs are regarded as the important suppressive immune cells in the tumor microenvironment, contributing to tumor progression and clinical failure of treatment with ICI [33]. By contrast, low levels of FOXP3+ Tregs could predict a benefit from immunotherapy. Our patient showed the presence of a poor FOXP3+ TIL density, which, coupled with the notion of high peripheral and intratumoral CD8+ cell infiltration, contributed to the decision to propose ICI therapy to this patient at the time of progression on afatinib.

While it is relatively common following treatment with an EGFR-TKI in EGFR-mutated 
NSCLCs with brain metastases, the brain response to systemic therapy such as ICI treatment may be impaired [33, 34]. Nevertheless, our group has previously reported an intracranial response of $55.5 \%$ in 9 patients with asymptomatic brain metastases and a PD-L1 score of $\geq 50 \%$ who were treated with upfront pembrolizumab in the absence of brain radiotherapy [35]. Therefore, even in the presence of asymptomatic brain metastases, ICI treatment should not be delayed, especially in the case of predictors of increased sensitivity, such as high PD-L1 expression.

\section{CONCLUSIONS}

In conclusion, we believe that proper patient selection for ICI therapy based on multiple immune biomarkers for a more personalized immunoscoring system could be warranted for EGFR-mutated NSCLCs with acquired resistance to an EGFR-TKI. Although barriers linked to cost as well as time necessary to return the test results may exist, it is the author's belief that immunoscoring based on multiple tests should be advocated in this clinical scenario. In the present case, we showed that a composite score based on an increase in TMB levels as well as elevated PD-L1 expression and simultaneous documentation of high CD8+/low FOXP3+ TIL density in the tumor microenvironment was able to identify an EGFR-mutated NSCLC patient with acquired resistance to an EGFR-TKI who experienced a complete and long-term response to ICI therapy.

\section{ACKNOWLEDGEMENTS}

We thank the patient for giving her written informed consent to publication of her case.

Funding. No funding or sponsorship was received for this study or publication of this article.

Authorship. All named authors meet the International Committee of Medical Journal Editors (ICMJE) criteria for authorship for this article and take the responsibility for the integrity of the work as a whole, and have given their approval for this version to be published.

Author Contributions. Sara Baglivo and Giulio Metro were responsible for the conception of the work, data collection and manuscript writing. Martina Mandarano, Sara Baglivo, Vienna Ludovini, Guido Bellezza, performed molecular analyses of the case. Matthias Joachim Fischer provided radiographic material. All authors read and gave their approval for the manuscript to be published in its final version.

Disclosures. Sara Baglivo, Martina Mandarano, Guido Bellezza, Vincenzo Minotti, Angelo Bonaiti, Matthias Joachim Fischer, Ilaria Birocchi, Fausto Roila, Niccolò Metelli, Vienna Ludovini and Giulio Metro confirm that they have no conflicts of interest to disclose.

Compliance with Ethics Guidelines. Written informed consent was provided from the patient for publishing her case in an anonymous form. A copy of the consent form is available for review by the editor.

Data Availability. All data generated or analysed during this study are included in this published article.

Open Access. This article is licensed under a Creative Commons Attribution-NonCommercial 4.0 International License, which permits any non-commercial use, sharing, adaptation, distribution and reproduction in any medium or format, as long as you give appropriate credit to the original author(s) and the source, provide a link to the Creative Commons licence, and indicate if changes were made. The images or other third party material in this article are included in the article's Creative Commons licence, unless indicated otherwise in a credit line to the material. If material is not included in the article's Creative Commons licence and your intended use is not permitted by statutory regulation or exceeds the permitted use, you will need to obtain permission directly from the copyright holder. To view a copy of this licence, 
visit http://creativecommons.org/licenses/bync/4.0/.

\section{REFERENCES}

1. The importance of detecting targetable EGFR mutations in NSCLC: know your exon. Available at: https://www.vumedi.com/video/the-importanceof-detecting-targetable-egfr-mutations-in-nsclcknow-your-exon/. Accessed 01 Nov 2021

2. To KKW, Fong W, Cho WCS. Immunotherapy in treating EGFR-mutant lung cancer: current challenges and new strategies. Front Oncol. 2021;11: 635007.

3. Lee CK, Man J, Lord S, et al. Checkpoint inhibitors in metastatic EGFR-mutated non-small cell lung cancer-a meta-analysis. J Thorac Oncol. 2017;12: 403-7.

4. Lee CK, Man J, Lord S, et al. Clinical and molecular characteristics associated with survival among patients treated with checkpoint inhibitors for advanced non-small cell lung carcinoma: a systematic review and meta-analysis. JAMA Oncol. 2018;4:210-6.

5. Mazieres J, Drilon A, Lusque A, et al. Immune checkpoint inhibitors for patients with advanced lung cancer and oncogenic driver alterations: results from the IMMUNOTARGET registry. Ann Oncol. 2019;30:1321-8.

6. Oxnard GR, Yang JC, Yu H, et al. TATTON: a multiarm, phase Ib trial of osimertinib combined with selumetinib, savolitinib, or durvalumab in EGFRmutant lung cancer. Ann Oncol. 2020;31:507-16.

7. Creelan BC, Yeh TC, Kim SW, et al. A phase 1 study of gefitinib combined with durvalumab in EGFR TKI-naive patients with EGFR mutation-positive locally advanced/metastatic non-small-cell lung cancer. Br J Cancer. 2021;124:383-90.

8. Yang JC, Gadgeel SM, Sequist LV, et al. Pembrolizumab in combination with erlotinib or gefitinib as first-line therapy for advanced NSCLC with sensitizing EGFR mutation. J Thorac Oncol. 2019;14:553-9.

9. Garon EB, Wolf B, Lisberg A, et al. Prior TKI therapy in NSCLC EGFR mutant patients associates with lack of response to anti-PD-1 treatment. J Thorac Oncol. 2015;10:S269.

10. Lisberg A, Cummings A, Goldman JW, et al. A Phase II study of pembrolizumab in EGFR-mutant, PD-
$\mathrm{L} 1+$, tyrosine kinase inhibitor naïve patients with advanced NSCLC. J Thorac Oncol. 2018;13: 1138-45.

11. Gettinger S, Rizvi NA, Chow LQ, et al. Nivolumab monotherapy for first-line treatment of advanced non-small-cell lung cancer. J Clin Oncol. 2016;34: 2980-7.

12. Peters S, Gettinger S, Johnson ML, et al. Phase II trial of atezolizumab as first-line or subsequent therapy for patients with programmed death-ligand 1-selected advanced non-small-cell lung cancer (BIRCH). J Clin Oncol. 2017;35:2781-9.

13. Garassino $\mathrm{MC}$, Cho $\mathrm{BC}$, Kim JH, Mazières J, et al. Durvalumab as third-line or later treatment for advanced non-small-cell lung cancer (ATLANTIC): an open-label, single-arm, phase 2 study. Lancet Oncol. 2018;19:521-36.

14. Hellmann MD, Rizvi NA, Goldman JW, et al. Nivolumab plus ipilimumab as first-line treatment for advanced non-small-cell lung cancer (CheckMate 012): results of an open-label, phase 1, multicohort study. Lancet Oncol. 2017;18:31-41.

15. Gubens MA, Sequist LV, Stevenson JP, et al. Pembrolizumab in combination with ipilimumab as second-line or later therapy for advanced nonsmall-cell lung cancer: KEYNOTE-021 cohorts D and H. Lung Cancer. 2019;130:59-66.

16. Luo J, Bandlamudi C, Ricciuti B, et al. Long-term responders to $\mathrm{PD}-1$ blockade in patients with advanced non-small cell lung cancer (NSCLC). J Clin Oncol. 2020;38:9549.

17. Schoenfeld AJ, Rizvi H, Bandlamudi C, et al. Clinical and molecular correlates of PD-L1 expression in patients with lung adenocarcinomas. Ann Oncol. 2020;31:599-608.

18. Lee SE, Kim YJ, Sung M, et al. Association with PDL1 expression and clinicopathological features in 1000 lung cancers: a large single-institution study of surgically resected lung cancers with a high prevalence of EGFR mutation. Int J Mol Sci. 2019;20:4794.

19. Toki MI, Mani N, Smithy JW, et al. Immune marker profiling and programmed death ligand 1 expression across NSCLC mutations. J Thorac Oncol. 2018;13:1884-96.

20. Peng S, Wang R, Zhang X, et al. EGFR-TKI resistance promotes immune escape in lung cancer via increased PD-L1 expression. Mol Cancer. 2019;18: 165.

21. Isomoto $\mathrm{K}$, Haratani $\mathrm{K}$, Hayashi $\mathrm{H}$, et al. Impact of EGFR-TKI treatment on the tumor immune 
microenvironment in EGFR mutation-positive nonsmall cell lung cancer. Clin Cancer Res. 2020;26: 2037-46.

22. Addeo A, Friedlaender A, Banna GL, Weiss GJ. TMB or not TMB as a biomarker: that is the question. Crit Rev Oncol Hematol. 2012;163:103374.

23. Chalmers ZR, Connelly CF, Fabrizio D, et al. Analysis of 100,000 human cancer genomes reveals the landscape of tumor mutational burden. Genome Med. 2017;9:34.

24. Carbone DP, Reck M, Paz-Ares L, et al. First-line nivolumab in stage IV or recurrent non-small-cell lung cancer. N Engl J Med. 2017;376:2415-26.

25. Hastings K, Yu HA, Wei W, et al. EGFR mutation subtypes and response to immune checkpoint blockade treatment in non-small-cell lung cancer. Ann Oncol. 2019;30:1311-20.

26. Wang X, Ricciuti B, Nguyen $T$, et al. Association between smoking history and tumor mutation burden in advanced non-small cell lung cancer. Cancer Res. 2021;81(9):2566-73.

27. Le X, Negrao MV, Reuben A, et al. Characterization of the immune landscape of EGFR-mutant NSCLC identifies CD73/adenosine pathway as a potential therapeutic target. J Thorac Oncol. 2021;16: 583-600.

28. Park LC, Rhee K, Kim WB, et al. Immunologic and clinical implications of CD73 expression in nonsmall cell lung cancer (NSCLC). J Clin Oncol. 2018; $15: 12050$.

29. Simoni $\mathrm{Y}$, Becht E, Fehlings $\mathrm{M}$, et al. Bystander CD8(+) T cells are abundant and phenotypically distinct in human tumour infiltrates. Nature. 2018;557:575-9.

30. Qiao M, Jiang T, Ren S, Zhou C. MA13.06: deciphering the characteristics of tumor microenvironment in EGFR-mutated non-small cell lung cancer. J Thorac Oncol. 2020;16:S182-3.

31. Dong ZY, Zhang JT, Liu SY, et al. EGFR mutation correlates with uninflamed phenotype and weak immunogenicity, causing impared response to PD-1 blockade in non-small cell lung cancer. Oncoimmunology. 2017;6:e1356145.

32. Galon J, Bruni D. Approaches to treat immune hot, altered and cold tumours with combination immunotherapies. Nat Rev Drug Discov. 2019;18: 197-218.

33. Navajas-Gonzales JM, Fan DD, Yang S, et al. The impact of Tregs on the anticancer immunity and the efficacy of immune checkpoint inhibitor therapies. Front Immunol. 2021;12:625783.

34. Metro G, Chiari R. Preface on "emerging treatment options for brain metastases from non-small cell lung cancer." Transl Lung Cancer Res. 2016;5: 561-2.

35. Metro G, Banna GL, Signorelli D, et al. Efficacy of pembrolizumab monotherapy in patients with or without brain metastases from advanced non-small cell lung cancer with a PD-L1 expression $\geq 50 \%$. J Immunother. 2020;43:299-306.

36. Metro G, Gili A, Signorelli D, et al. Upfront pembrolizumab as an effective treatment start in patients with PD-L1 $\geq 50 \%$ non-oncogene addicted non-small cell lung cancer and asymptomatic brain metastases: an exploratory analysis. Clin Transl Oncol. 2021;23:1818-26. 